\title{
Decision-making within missing person search
}

\author{
Kyle Harrington ${ }^{1}\left[\right.$. Michael Brown ${ }^{1} \cdot$ James Pinchin $^{2} \cdot$ Sarah Sharples $^{1}$
}

Received: 14 November 2017 / Accepted: 13 August 2018 / Published online: 30 August 2018

(c) The Author(s) 2018

\begin{abstract}
This paper reports the findings of a series of interviews with search and rescue volunteers. Participants were asked to recall accounts of particular incidents which involved searching for a missing adult who could be considered 'vulnerable'. The purpose of this study was to discover what types of decisions are made during missing incidents; including a consideration of the factors which affect these decisions and the main focuses of attention throughout the incident. Such an understanding may help to shed light on best practices which could inform decision-making support tools for families of the missing and identify the user-requirements of a future technology designed to help find missing people. Interviews were conducted using the critical decision method (CDM) to elicit specific information about the decisions and challenges faced by search and rescue teams during missing person searches. Critical decision points were identified and sequenced for each incident. Emergent thematic analysis (EMA) was applied to the transcripts to identify themes across various incidents; these themes were explored in detail using a mixed-method approach. This study builds upon the methodological approach of CDM using a two-tiered approach to analysis which seeks to discover the focus of practitioners' attention as they progress through missing person searches. A decision-sequence diagram was created to clearly show the sequence of each decision and trends across all incidents; a table was produced to show the relative importance of each aspect across decisions. Finally, strengths and weaknesses of this approach to incident analysis are discussed.
\end{abstract}

Keywords Critical decision method $\cdot$ Cognitive task analysis $\cdot$ Search and rescue $\cdot$ Incident analysis $\cdot$ Emergent thematic analysis · Interview

\section{Introduction}

Every year the police receive around a quarter of a million reports of missing people in England and Wales alone (National Crime Agency 2016). Whilst the vast majority of missing people return home safely, around a third of individuals suggested to police they felt in danger at some point (Biehal 2003). It is estimated that up to $80 \%$ of adults who go missing also have mental health issues, thus increasing the risk to their own safety (Missing Persons Data Report 2014-2015). People with care and support needs and others who could be considered 'vulnerable' are much more likely to go missing than the general population and are at much greater risk of injury or fatality if they do (Perkins 2013).

Kyle Harrington

kyle.harrington@nottingham.ac.uk

1 Human Factors Research Group, University of Nottingham, Nottingham, UK

2 Horizon, University of Nottingham, Nottingham, UK
Dementia patients alone constitute over $15 \%$ of all search and rescue operations in England and Wales yet only represent around 1\% of the population (Prince et al. 2014). Not only is the prevalence of vulnerable adults who go missing disconcerting, but so too are the associated risks. The survival rates of 'vulnerable' adults who go missing (including 'despondents', those with cognitive impairments, mental illness and/or drug dependencies) are reported to be much lower than that of the wider population.

In a retrospective study, Koester analysed the database of the Virginia Department of Emergency Services records of search and rescue (Koester and Stooksbury 1995). It was discovered that people living with Dementia who become missing were usually found within a mile of the position they were last seen ( $89 \%$ within a mile, mean 0.6 miles, median 0.6 miles, range 2 miles). All of those living with Dementia that were found within $24 \mathrm{~h}$ survived, but mortality rates increased to $54 \%$ after this critical period.

Koester and Stooksbury (1995) found some evidence to support the notion that people living with dementia take 
the path of least resistance when they abscond or elope, with most of those found on or close to roads; many in large bushes or briars and a large amount also in creeks or drainage systems. It is also reported that dementia patients are generally unresponsive to assistance even when uninjured and will rarely leave physical clues as to their location. This further emphasises the importance of understanding the search and rescue task in detail to offer guidance to search and rescue professionals and the families of the missing. Furthermore, by understanding how such incidents unfold, new technologies can be developed to better address the needs of those affected.

Perhaps the largest area of research focusing on search behaviour has been developed from a perspective influenced heavily by cognitive psychology (Wolfe 1994). Typically, visual search experiments have been employed which involve participants attempting to find a target item amongst a complex array of distractor items in a controlled environment. It is common during this task to vary the properties of distractor and target items and to measure either reaction times or task accuracy. It is also common for other measures to be taken, including FMRi (Ellison et al. 2004) and electrophysiological measures (Kiss et al. 2007). Visual search experiments shed light on an important, but ultimately very narrow aspect of search activity in a very controlled environment and as such may not necessarily be directly applicable to real-world search and rescue operations which involve the allocation of tasks in a team environment and inferences about the psychological dispositions of the missing person.

Another area that investigates search activity is search theory which was originally developed by the US military to efficiently search for enemy submarines (Koopman 1946). Research of this nature involves mathematical modelling of abstract agents searching in space to find a target where each search agent is considered to have a probability of detection over a certain range. Given a certain probability of detection, optimal "sweep width estimation" (the distance between two separate search agents or separate passes) can be obtained. Through the use of this modelling technique, various methods for efficiently searching have been developed and are often used as the basis for search and rescue operational manuals (Cooper et al. 2008). However, these models are heavily prescriptive and do not describe how search and rescue operations are actually conducted as they do not account for human actions, geographical restrictions and contextual clues which may inform the search parameters.

A relatively small number of research studies have taken a more holistic approach which attempts to better understand the context in which missing incidents occur. For example, the missing person conceptual model, proposed by Rowe et al., (2015) posits an interplay between the contextual, situational and neurological antecedents that result in a person leaving a place of safety and becoming missing. The model is excellent for sketching the precursors of missing incidents and highlighting some of the consequences of such events, but does little to elucidate activities during missing incidents themselves. Conversely, there is also work investigating the longer term impact of missing incidents; including the effect on the families of the missing (Lois 2001; Parr et al. 2015; Stevenson et al. 2013). Lois (2001), for example, investigated how search and rescue volunteers manage the intense emotions of victims' and families during missing incidents and how this led to close relationships being formed between families of the missing and volunteer search and rescue groups.

These typically more ethnographic approaches allow for a greater understanding of the context in which missing incidents occur, but provide relatively little insight about how individuals respond to the task demands of missing incidents. Task analysis, on the other hand, is a series of techniques which follow a structured approach for the purposes of understanding the scope of a particular task and how practitioners navigate decision space to achieve their goals. Task analysis refers to a diverse range of tools which are often employed within the human factor discipline to describe in detail various aspects of operator behaviour and also to provide a comprehensive description of operator tasks. Task analysis techniques are often used to identify sources of error in complex socio-technical systems and ultimately suggest ways in which the system could be improved (Shepherd 2000; Wilson and Corlett 2005).

Methods such as these can be used to discover various subtasks as well as the ways in which these are distributed in time and space and between various team member task analysis techniques have also been adopted previously to facilitate the use of unmanned aerial vehicles for search and rescue operations (Adams et al. 2007) and to understand the distributed situational awareness of aerial search and rescue operators (Plant and Stanton 2016).

Traditional hierarchical task analysis techniques tend to be action-oriented approaches and focus on providing a description of the observable aspects of operator behaviour (Stanton 2006). Typically, hierarchical task analysis involves adopting a top-down approach organise tasks and subtasks into appropriate organisational hierarchies. In this way, Hierarchical task analysis can be used to identify potential sources of error and inefficiency as well as define potential operational constraints to improve future systems or to provide training documents (Shepherd 2000).

However, hierarchical task analysis techniques are typically focused on the goals of operators and not well suited to elicit underlying cognitive and affective states that give rise to differing courses of action (Salmon et al. 2010). An alternative set of approaches known collectively as cognitive tasks analysis, provide methods to overcome some of the traditional shortcomings of hierarchical task analysis 
"yield information about the knowledge, thought processes and goal structures that underlie observable task performance" (Schraagen et al. 2000, p. 3) and as such can be used to discover descriptions of both overt behaviours and covert cognitive functions for complex tasks that last several hours or days (Clark et al. 2008). Militello and Hutton (1998) highlight the increasing need for cognitive task analysis particularity in environments which require inference, diagnosis, judgement and decision-making. As such, cognitive task analysis approaches are preferable to more traditional hierarchical task analysis techniques in domains where experts may find the justification for their decisions difficult to verbalise.

The critical decision method (CDM) (Klein et al. 1989) is one such method. CDM is an incident-based knowledge elicitation technique based on the critical incident technique (Flanagan 1954) designed to uncover the decision-making processes of experts. CDM allows participants to define the scope of the problem in their own terms and as such not only provides scope for the analysis of the allocation and distribution of subtasks but also allows for the possibility of creating alternative solutions. It consists of semi-structured interviews which require the participant to give an account of a previous incident. After which, the interviewer and participant construct a timeline of events and identify so-called "Critical Decisions". Finally, for each decision point, the interviewer asks a series of probing questions, designed to elicit specific information about each decision within the context in which that decision was made.

Klein (1993) proposed the theory of recognition-primed decision-making (RPD) to understand the decision-making capabilities of experts. Klein calls his general approach, "naturalistic decision making" (Salas and Klein 2001; Zsambok and Klein 1997), which can be broadly characterised as the study of decision-making in real-world, naturally occurring environments. The naturalistic decision-making approach is particularly suited to uncertain dynamic environments which feature ill-structured problems, time stress and high stakes (Chaudet et al. 2015). Klein describes how he came to study the rapid responses of firefighters and their ability to make rapid and effective decisions in complex environments (Klein et al. 1988) which he used to inform his RPD model. The RPD postulates that in time-critical situations, experts do not often have time to deliberate between numerous possible courses of action. Instead, when an expert is confronted with a novel situation, they rely on their experience and access their memory of similar situations to decide on an appropriate course of action. The RPD asserts that expert practitioners will attempt to find examples from their memory which bear resemblance to the current situation in the hopes of choosing a suitable course of action. They will then use mental simulation to evaluate whether the course of action is satisfactory, amending and revising their plans if necessary. Klein suggests that the RPD allows for rapid and effectual decision-making in situations where conscious deliberation would be too slow (Klein 1993). Rather than trying to arrive at optimal solutions, experts, it is suggested, use cues to arrive at merely satisficing courses of action. This 'intuitive' ability of experts has been elaborated by Okoli et al. (2016), who suggest that experts not only have a broader body of knowledge and experience from which to draw from, but that they have also developed an understanding of which cues should be attended to and which should be ignored. It is suggested that this ability to effectively 'filter' information and identify principle cues reduces the strain on working memory and allows practitioners to devote more cognitive resources to only those aspects which are immediately relevant to decision-making (Okoli et al. 2016). The differences in strategic approaches employed by experts and non-experts in the domain of missing person searches have been confirmed by Rogalski (1999).

However, other researchers investigating decision-making appear more sceptical about human decision-making ability. Kahneman (1979), points to numerous examples where various cognitive biases negatively affect people's ability to make "rational" choices. Kahneman and Tversky (1979) suggest that forecasts and predictions are often likely to be wrong, owing to the fact that people often overestimate the probability of particularly salient events, whilst underestimating much more mundane outcome; this bias, known as the availability heuristic, is thought to be pervasive in judgement and decision-making. Other similar biases identified by Kahneman and Tversky include the representativeness heuristic and a failure to consider the base rate; the average frequency at which an event is likely to occur (Tversky and Kahneman 1973). The representative heuristic is usually employed when people are asked if one object or event belongs to a particular class or process. However, rather than using prescriptive rules and analytical reasoning to determine whether this is the case, people make their judgements often by considering mere superficial similarity or rely on stereotypes.

Additional research also illustrates that people avoid losses much more than they seek gains. 'Loss aversion', demonstrates that people are not always strictly judicious when evaluating gains and losses. They do not view forgone gains in the same manner as losses (Thaler et al. 1997). Such examples call into question the effectiveness of expert decision-making suggested by Klein, and more fundamentally; challenge the idea that decisions are made by fully-informed, self-interested rational agents trying to maximise a certain utility function (Kahneman and Tversky 1979).

Gigerenzer and Goldstein (1996) suggest that humans make inferences about their environment under conditions with limited time and knowledge, and therefore, do not always have unlimited time, knowledge or resources at their 
disposal to make entirely 'rational' decisions. Instead, they demonstrate through the use of a computer simulation, that in many cases merely satisficing solutions outperform multiple regression inferences in terms of speed and accuracy. This is taken to show that cognitive mechanisms capable of successful performance need not be derived from an exhaustive consideration of all possible options.

Smith and Kida (1991) have argued that the typical experimental setup in which certain heuristics and cognitive biases are tested are often very artificial and do not adequately represent the context in which expert decisionmaking occurs, calling into question the generalisability and ecological validity of such studies. Though the authors confirm that expert practitioners are not immune to cognitive biases, they argue that many of these biases are mitigated or modified in studies which used experts performing familiar tasks. The prevalence of systematic biases resulting from a dependency on heuristics should not necessarily be taken to suggest that heuristics are inherently 'bad' or that they will more often than not result in sub-optimal performance. Heuristics can be considered adaptive strategies which evolved in tandem with other fundamental psychological mechanisms (Goldstein and Gigerenzer 2002), and therefore, must have been selected for. It has been argued that heuristics are used as a cognitive method for reducing the complexity of decision-making in environments which are ambiguous, time pressured or cognitively overloading (van den Heuvel et al. 2014). The survivability of such mechanisms demonstrates that heuristics must have been beneficial overall to the species, but it should not be surprising that these generalisations may be sub-optimal in marginal, or otherwise scientifically contrived cases.

Challenging, complex events such as missing person searches, require focused attention and for this reason are also much more likely to be vividly recalled than routine events (Crandall et al. 2006). Typically, CDM is used to better understand decision-making in complex real-world environments to extract tacit information from experts for the purposes of designing training manuals, expert systems or operator interfaces. One of the suggested applications for knowledge elicitation from this approach is the development of new systems (Crandall et al. 2006; Klein et al. 1989; O'Hare et al. 1998). CDM has also been used to identify user-requirements for the development of new technologies in complex socio-technical systems with associated timecritical tasks such as emergency medical dispatch (O'Hare et al. 1998; Wong and Blandford 2002). Identifying cues and decisions used by experts may also help to discover a deeper understanding of the best current practice of missing person search. In turn, this information may help to inform the guidance given to families of the recently missing, or to develop decision-making support software to that end.

\section{Method}

\subsection{Participants}

Five participants were selected from three search and rescue organisations within the UK, including associated members of mountain rescue and low land rescue teams. Potential participants were identified during an initial consultation with a subject matter expert and senior mountain rescue volunteer; this initial consultation also allowed the interviewer to familiarise himself with the general operations, terminology and command structure employed within search and rescue teams. Each participant had at least 5 years search and rescue experience and was at least 'team leader' (or equivalent) within their respected organisation. Whilst the sample size in this study is small, similar research into decision-making within search and rescue has involved fewer interviewees (Plant and Stanton 2016). It also should be noted that, these participants only represent UK search and rescue teams, it is likely that search and rescue operations in other countries vary in terms of their duties, responsibilities and communications with other organisations.

\subsection{Ethics}

Participants were fully informed to the purpose and nature of the study and signed consent forms prior to the interviews. Participants were made aware of their right to withdraw at any point and all names and identifying information were removed from the transcripts, to ensure confidentiality. The data was stored securely on a password-protected computer, in accordance with the University of Nottingham's data policy. Ethical approval for this study was granted by the University of Nottingham's Ethics committee within the faculty of engineering.

\subsection{Interview technique}

Participants were interviewed using CDM (Crandall et al. 2006). Interviews lasted approximately one and a half hours, with the shortest interview lasting approximately $1 \mathrm{~h}$ and 15 and the longest, one and three quarter hours. This variability is in part due to the semi-structured nature of the interviews and individual differences between participants and incidents. During the course of the interview participants were asked to recall in detail a single incident from their own personal experience which involved looking for an adult who could be considered 'vulnerable'. As participants recalled a particular incident, the interviewer allowed the interviewee to retell the incident with minimal interference, and constructed a timeline of the event. A secondary discussion of the incident involved the researcher recalling his record of 
the incident to the participant; verifying and amending this record on the advice of the participant. This stage ensured that the salient facts of the incident were understood by both parties, and also allowed for a verification of the correct sequence of the events. This was an important stage, as many participants recalled incidents somewhat anachronistically, which sometimes led to confusion about the order in which events took place. During the next stage of the interview 'Critical Decisions' were identified collaboratively between the interviewee and the interviewer. Participants were told that a 'Critical Decision' was a decision that required expertise and/or judgement rather than a routine and standardised operating procedure. Additionally, they were told a 'Critical Decision' might be a point in the incident in which somebody with less experience would have done differently. The interviewer and interviewee then discussed and agreed upon which decisions within the incident were 'Critical Decisions'.

Once the critical decisions had been identified, the researcher asked a series of standardised probing questions for each decision point in an attempt to further understand the decision-making process behind each decision, revisiting and clarifying the incident in the process. This stage was similar to the "deepening sweep", suggested by Crandall et al. (2006). Whilst there are some suggested categories and probing questions listed in Chap. 5 of 'Working Minds' (Crandall et al. 2006), it is advised that these should be modified to suit the current purpose of the study. In the current study, the areas of particular interest are, affective states, cues, decisions and effects. As such, a series of standardised probing questions were asked during a 'deepening sweep' to elicit information about each area of interest. A script was written for the purpose of the interviews, which included a briefing as well as prompts and probing questions (Table 1) which were adapted from Crandall et al. (2006).

These probing questions were developed to better understand what types of decisions were made, the cues and other factors which informed those decisions, the consequences of the decisions and the role (if any) that emotion might play in influencing decision-making within high stakes critical incidents.

\subsection{Emergent thematic analysis}

CDM can elicit knowledge and information about specific incidents and expertise of professionals, but data extracted from the interviews requires further analysis and representation to identify commonalities across incidents. ETA has been applied to CDM interviews to identify overall decision strategies and broad categories of behaviour as well as further describe the context in which the task occurs (Wong and Blandford 2002, Wong and Blandford 2004). ETA can be used to distil CDM transcripts, thus enabling broad themes to be identified and analysed without committing oneself to any a priori assumptions. This preserves the exploratory nature of $\mathrm{CDM}$ whilst increasing yield and ensuring analytical rigour.

The interviews and handwritten timelines were transcribed. Excerpts referring to critical decisions were coded in the transcripts. Once these critical decisions were abstracted from the data, sub-themes were identified for each decision in a secondary stage of analysis. This involved further dividing each of the critical decision excerpts into smaller sub-sets, representing decision-specific themes,
Table 1 Probing questions

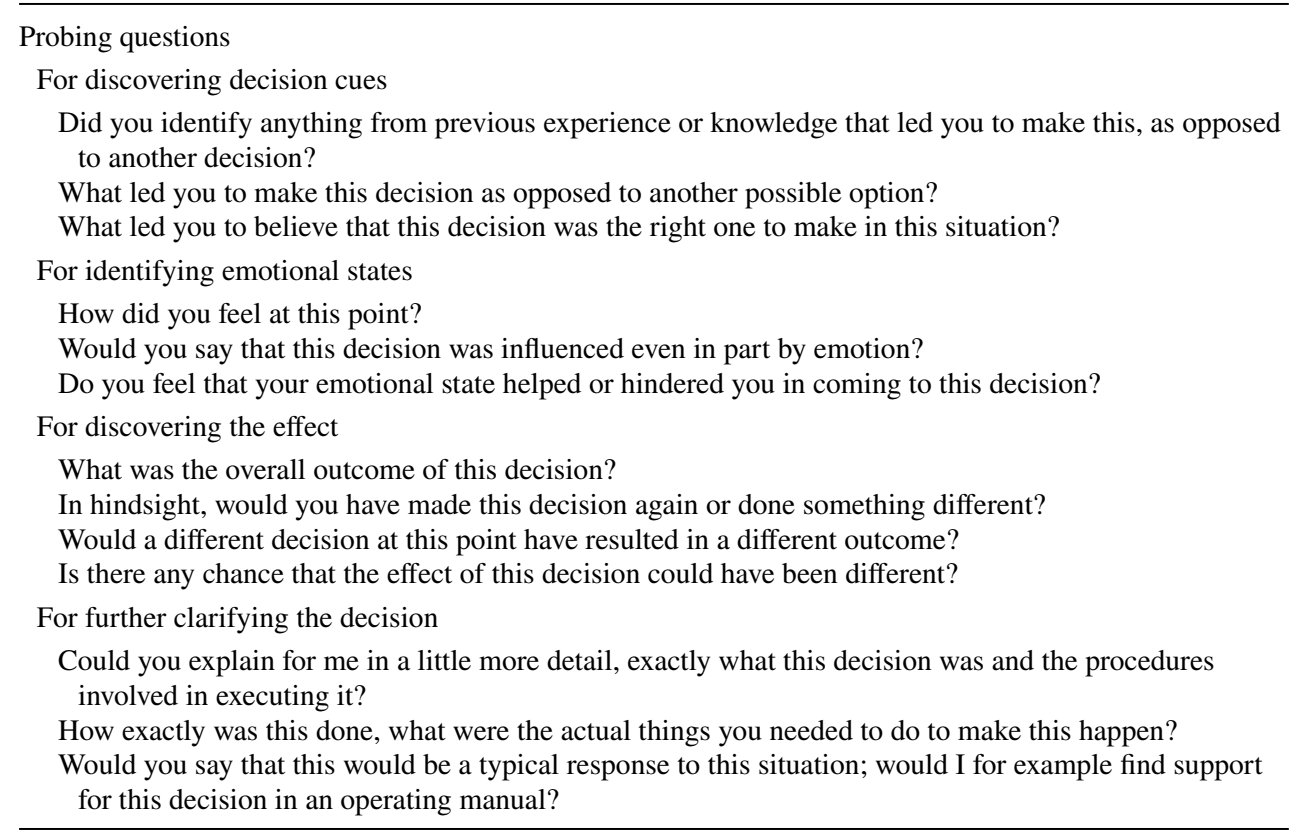


and further elucidated the main considerations during each decision. The sub-themes of each critical decision are given in "Appendix 1", Table 3, in the column, "Inclusion Criteria and Sub-Themes", alongside a figure which indicates the total number of references made that were within the sub-theme.

Additionally, an entirely separate coding scheme was created to identify considerations which were pervasive throughout the entire incident. These "Global Themes" included "Team Safety, Wellbeing and Expectation Management", "Equipment", "Communications with the Police" and "Clues", which were evident to some extent across every decision. This additional stage of coding allowed for an exploration of where attention was focused throughout the incident and also allowed for a "cross-referencing" technique which demonstrated the relative importance of each of these factors within incidents. This "cross-referencing" technique was performed in N-Vivo, using the "Matrix Tabulation" function and identified excerpts from the transcripts which were common to both a given critical decision and a particular global theme.

The two types of coding schemes (critical decisions and global themes), loosely correspond to Braun and Clarke's distinction between "Inductive and Theoretical Thematic Analysis" (Braun and Clarke 2006). Where the identification of critical decision points is largely a 'top-down' and 'Theoretical' process; due to the critical decision points being explicitly identified prior to the analysis. Contrastingly, the identification of 'Global Themes' is far more bottom-up, 'Inductive' and data-driven, as no themes were identified prior to the analysis nor was coding forced into a pre-existing coding scheme. These two approaches in conjunction allow for operational decision points to be identified which can be clearly understood by practitioners whilst allowing for deeper, often more subtle themes to emerge. Performing the more 'theoretical/top-down' method of analysis first also provides some guidance as to what themes are common across multiple decision points and structures the data before a more in-depth coding scheme is generated.

This differs somewhat from the approach adopted by Wong and Blandford (Wong and Blandford 2002, Blandford and Wong 2004), as the critical decisions which were identified during the interviews were the basis of the initial coding themes. The approach taken here also extends upon the ETA approach by identifying various sub-themes for each of the critical decisions, as well as global themes which pervade incidents. A distinct advantage of this approach is that using the cross-referencing technique, the relative importance of specific issues can be identified for each of the critical decisions.

\subsection{Representation}

Once coding was complete, a diagrammatic representation of the search task was produced to show the temporal sequence in which decisions were made. Whilst there are many methods of task representation within incident and accident analysis, none of these seemed wholly appropriate for representing the data elicited from the interviews. A popular approach to incident analysis is Rasmussen's Accimap (Rasmussen 1997), which looks at the contributing factors leading to a specific accident. However, this was deemed inappropriate on multiple counts. First, without significant modification, the Accimap is designed to analyse only one specific incident rather than identifying trends across multiple similar incidents (Salmon et al. 2012). Second, the Accimap shows how various factors across multiple levels all contribute to an unintended and accidental consequence. This differs from the incidents under investigation in that, the search task is an attempt to mitigate and resolve an unfavourable situation rather than an analysis which seeks to understand why these unfavourable states of affairs arose.

An incident representation diagram was produced specifically for the purposes of representing the relative importance of themes as they relate to each critical decision (Fig. 1). The diagram resembles the original timelines created during the interviews but uses generalised decision themes rather than the specific decisions reported in each interview. The diagram also shows the sub-themes extracted for each decision point as well as global themes salient to each decision.

\section{Results}

Decision-making within the search and rescue context, whilst dynamic; typically followed an ordered sequence in all of the incidents described by participants. There is a great deal of variability in the specifics of each decision, for example 'decide search area' was a decision made in every incident, but the details of where to search were dependent upon environment, context, available resources, experience of the incident commander(s) and knowledge of the missing person. During the analysis stage decisions across interviews were grouped into decision "themes" and seven critical decisions emerged; "Decide if callout valid", "Teams and Additional Resources", "Plan Search Area", "Sectors and Sections", "On-Ground Decisions", "Care of Casualty" and "Abandon/Stand-down". A full description of these decisions is given in "Appendix 1", Table 3, which also shows the inclusion criteria for each decision category, as well as examples of transcript excerpts which give an indication of what each respective decision entails. Further analysis was conducted to further elucidate specific considerations during each decision, this analysis was conducted by sorting 


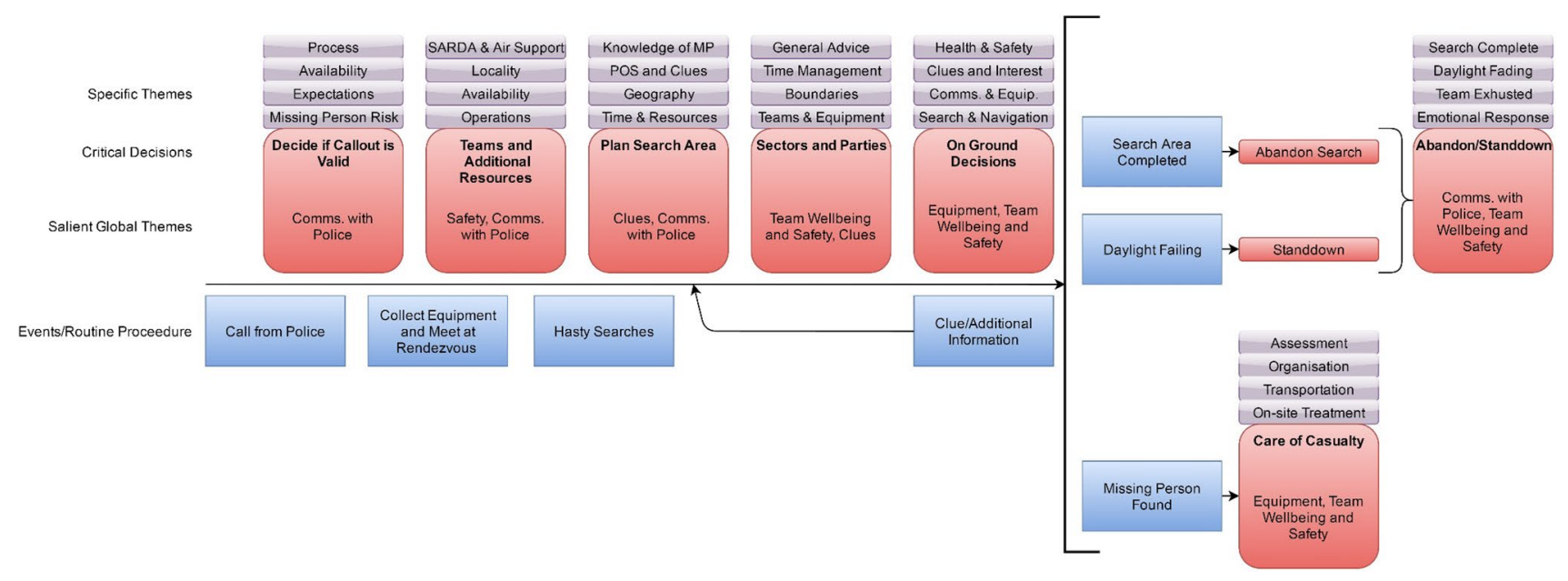

Fig. 1 Critical decision sequence diagram

excerpts relating to each decision into sub-categories, which are included in the "Inclusion Criteria and Sub-Themes" column in "Appendix 1", Table 3.

The thematic category "On-Ground Decisions", does not represent a single decision point identified by participants, but instead represents a general grouping of the less frequent and more variable responses to environmental and contextual factors faced by 'on-the-ground' teams. Whilst no particular interviewee identified these specific decisions as 'Critical', it was decided that for completeness these important but less predictable decisions should form part of the task analysis.

Once the decisions which had been identified within the interviews had been coded within the transcripts, a more general and pervasive coding scheme was constructed to identify domain-specific considerations of principle decision makers as they worked through each stage of an incident. The four "Global Themes" were; "Team Safety and Wellbeing", "Equipment", "Communications with Police" and "Clues". A full description of the findings, as well as supporting quotes can be found in "Appendix 2", Table 4. Numbers presented within both Tables 3 and 4 refer to the total numbers of references made for each theme, or sub-theme.

\subsection{Intersection of global themes and critical decisions}

Table 2 shows the number of references that were common to both the critical decisions and the global themes (both full and partial matches), after the cross-referencing technique was performed in N-Vivo. Whilst these results should be taken with caution, Table 2 gives an indication of the relative importance of each global theme to each critical decision.

\subsection{Critical decision sequences}

Figure 1 shows the generalised timeline of events and decisions from the perspective of search and rescue teams during missing incidents. This timeline was constructed by an aggregate consideration of the typical decision-sequence order that was described during the interviews and further verified by one of the interviewees as being generally representative of missing incidents.
Table 2 Cross-referencing of decisions and global themes

\begin{tabular}{llllr}
\hline & $\begin{array}{l}\text { Team safety and } \\
\text { wellbeing }\end{array}$ & Equipment & $\begin{array}{l}\text { Communications } \\
\text { with police }\end{array}$ & Clues \\
\hline 1: Is callout valid & 6 & 2 & 13 & 0 \\
2: Teams and additional resources & 7 & 5 & 11 & 1 \\
3: Plan search area & 12 & 2 & 14 & 13 \\
4: Sections and parties & 7 & 4 & 13 & 7 \\
5: On-ground decisions & 8 & 4 & 8 & 2 \\
5: Care of casualty & 1 & 9 & 8 & 0 \\
6: Standdown & 8 & 0 & 1 & 2 \\
\hline
\end{tabular}




\subsection{Comments on results}

Search and rescue operations typically followed a standardised and predictable sequence of decisions, though the specific decision outcomes arrived upon were entirely dependent upon the circumstances of the search. Decision-makers apply a broad set of heuristics to the search task but often have to improvise depending upon the information they have available to them about the missing person, the environment of the search, and the resources at their disposal. Expert search and rescue practitioners describe their decision-making process as a mixture of 'Expertise and Judgement', rather than strict adherence to a rigid protocol. Despite this fact, decision-makers largely made the similar decisions in the same order in each case and there was a great deal of commonality in the decision-making process across incidents.

Police involvement was reported to be generally high (see Table 4), though there were differences between respective search and rescue associations and local Police organisations, this was shown to impact both the number and scope of decisions made by search and rescue teams. In two of the five interviews conducted, search and rescue volunteers reported that Police effectively managed the overall search plan, decided the search area, and ultimately issued the order to 'stand-down' as appropriate. The other three participants reported a much higher degree of autonomy in the decision-making process, although even in these cases they worked very closely with the local police. In a few cases, this involved the search and rescue teams requesting background checks or asking for additional resources (such as support from the Royal Air Force) to be called in. The importance of this communication is reflected in Table 2, in which communications with the police features as the most salient global theme in all but the "Care of Casualty" decision point.

Decision-makers within search and rescue teams described four separate planning stages which occurred before any formal search activity occurred (Fig. 1) and this was taken as evidence that search and rescue experts viewed time as a resource which needed to be allocated appropriately. Search and rescue controllers and other decisionmakers tended to plan their searches with the knowledge that teams may be conducting the search for up to several days, and therefore, spent time planning how the use of their resources could be maximised over several hours. Incident controllers and other decision-makers ensured that they and their teams had appropriate provisions prior to the commencement of any given search and acted under the assumption that adequate preparation would lead to more effective searches. Search plans were reported to be informally constructed immediately following the callout. Incident commanders often had a general idea of the overall search plan upon arriving on scene and would continue to revise and clarify their plan dependent upon the resources that were likely to be available up until the moment of deployment.

Areas of high probability or those which required a lower amount of resource commitment were usually searched first during a preliminary "hasty search"; this involved the search teams looking across footpaths and accessible areas before a more formal and structured approach to search began. Hasty searches are a preliminary sweep of the area which utilise available personnel resources whilst team members are still arriving on scene. Once an initial hasty search had been conducted, teams were split into smaller groups of three to five people and allocated a clearly defined area, preferably with a natural boundary radiating out from the position of last sighting. However, no interviewees considered "hasty searches", to be a "critical decision", instead they considered this informal act of searching to be a standard procedure, requiring little deliberation or coordination and helping to maximise the time and resources at their disposal before a more thorough plan could be actioned.

Generally, decision-makers tended to be forward thinking and strategic but also responded well to new information. Search and rescue organisations have to adapt if their assessment of the situation changes due to external factors such as additional information from the police, or if one of the search teams finds a clue. In this case, teams will usually be asked to hold their position, and may be called back for redeployment whilst the search plan and (if necessary) position of last sighting is revised. Such an event occurred in two of the incidents described by interviewees, and is represented in Fig. 1. In both of the incidents described, decision-makers revised their search area on a new assumed position of last sighting.

When asked about the effect that emotion might have upon decision-making, interviewees seemed to indicate taking a professional attitude towards the search task and maintained emotional distance from the act of searching (e.g.), "You don't have time to wear your heart on your sleeve" [P3]. This emotional distance may be due to the fact that all of the interviewees had been involved in numerous cases with negative outcomes, and therefore, had realistic expectations. Direct questioning, therefore, may not be the best way to understand the role that emotion plays in influencing the decisions of professionals within this context.

Managing team expectations was considered to be an important role of incident commanders and team leaders. One incident commander reported not telling his team he thought it was very unlikely the person they were looking for was still alive to maintain team morale; "You don't tell em that, you keep it to yourself.-The lads, they know how many beans make five, but they still keep searching, they don't pack up until they're told" [P3]. Managing Team Expectations, Safety and Wellbeing was identified as a "Global Theme", given in Table 4. However, it was not 
possible to entirely separate "Managing Team Expectations" from managing other aspects of team safety and wellbeing and preserve the principle of exclusivity as described by Braun and Clarke (2006), as interviewees would often speak in general terms about their team's physical safety and psychological wellbeing.

\section{Discussion}

CDM alone provides an excellent framework for understanding expert behaviour across difficult or challenging incidents and provides a clear framework for conducting semi-structured interviews. Additionally, when ETA is performed on interview data greater detail and understanding of the operator task can be obtained. Wong and Blandford (2002) highlight the three major strengths of this approach. First, it "promotes the exploratory nature of the CDM", making it an excellent research methodology for a little understood problem. Second, it "Balances Emergence and Theory", this was particularly true of the study in question, in which critical decisions were identified in conjunction with the interviewees, but 'Global Themes', were only identified through an iterative and emergent process. Finally, this method is fast and practical. Whilst combining the CDM with ETA is more time consuming than the CDM alone, it yields a much greater insight and has the flexibility to focus on specific aspects of interest, in this case the domain-specific considerations of decision-makers engaged in missing person search.

When asked about the emotional states practitioners experienced during the search ("How were you feeling at this point?"), search practitioners would often report on their hypotheses about the missing person rather than provide any insight on the level of emotional arousal caused by the difficulty and importance of missing person search. Through clarification, practitioners did sometimes state how they were feeling at the time, but this was usually an operational consideration regarding the likelihood of a positive outcome. This suggests that whilst it is possible to discover some of the affective states experienced during a stressful and time-critical incident, direct questioning during retrospective accounts may not be the best approach for expert practitioners. Luini and Marucci (2015) used the framework of naturalistic decision-making to develop an approach for understanding the influence of emotions on cognitive processes. However, their approach comprised of a series of experimental trials investigating the reaction times and emotional responses of participants' performance in a firing range, and therefore, despite the fact the study could broadly be described as "Naturalistic Decision Making", their methods for discovering the role of emotion in cognition are not applicable here. An alternative approach might involve direct observation of incidents, or the application of discourse analysis techniques on the transcripts.

The decision-making process described by the search and rescue experts seemed to concur with Klein's description of naturalistic decision-making, specifically; the recognitionprimed decision model (Klein 1993). Klein suggests that rather than weighing up all potential courses of action and deciding upon the most optimal, decision-makers instead use prior experience to quickly categorise situations and apply learned schemata to decide upon an appropriate yet merely satisficing course of action. Klein also suggests that once a potential course of action is under consideration decisionmakers run a mental simulation to evaluate how successful that decision would be within the context of the current situation, revising and adapting if necessary (Klein 1993).

A further benefit to this approach was discovered during the analysis stage. Coding for 'Critical Decisions' before the 'Global Themes' allows the analyst to become very well acquainted with the interview data before attempting to identify broader themes which pervade across decision points.

Similar to the approach adopted by Wong and Blandford (2002), sub-themes were also identified within the critical decision points. Coding for the sub-themes was conducted after all the data extracts for the critical decision points had been fully coded in all instances in which they appeared in the data set. Introducing a second level of coding within the critical decision points allowed for a more specific and detailed account of each decision and which aspects practitioners deemed important.

Combining these various approaches across multiple levels of analysis created a sophisticated dataset. Using database software such as N-Vivo allowed for insightful results to be returned when presented with a variety of queries. When coded interview data is formatted in this way, it is possible to return all data items that relate to both a specific decision point and a specific global theme. For example, one may wish to find specific excerpts that relate to "Team Safety and Wellbeing" within the "Plan Search Area" decision to understand what safety considerations are made by team leaders when initially planning a search.

A potential limitation of this study, was the small sample size. However, Saunders et al. (2018) suggests that saturation should be operationalised in a way that is consistent with the research question and analytic framework adopted. No new critical decisions were identified after the second interview, which could be taken to indicate data saturation was reached. There was also considerable consistency between the decisions identified and the general order in which decisions were made. However, there were differences in the levels of responsibilities between different Search and Rescue associations and these differences may vary even more greatly between countries. Whilst the decisions identified for the search and rescue volunteers may be considered 
broadly reflective of the practices of search and rescue teams in the United Kingdom, there may be substantial differences in the procedures and considerations of search and rescue teams in different countries with different climates and legal systems.

\section{Conclusion}

This study attempted to discover what types of decisions search and rescue teams make during missing incidents and identify the factors which influence these decisions. Seven such decisions were identified, along with four global themes, which indicate the focus of attention throughout the incident. The results demonstrate the effectiveness of using the CDM combined with ETA. Furthermore, the introduction of a two-tiered coding scheme during the analysis also highlighted which particular aspects and considerations were important at each stage of the search process, and therefore, indicated the main focus of practitioners' attention as they conducted a task. This approach expands upon the CDM and has the potential to be applied to a wide variety of incident analysis studies. However, efforts to elicit the affective states of expert practitioners during an operation were of limited value. This work forms the basis of a user-requirements analysis for technology that may be able to assist in missing person search and highlights the decision-making processes of experts through various stages of missing person search. Future work will use a similar methodology to investigate the decision-making processes of familial and professional carers during missing incidents; both to compare and contrast decision-making between expert and non-expert groups and to better understand information exchange between the families of the missing and search and rescue volunteers.

Acknowledgements The authors would like to thank Mountain Rescue England and Wales and the Association of Lowland Search and Rescue for their cooperation and expertise during this study. This work is part of an ICase Studentship (13220154) sponsored by the EPSRC and Philips Research, and supported by the RCUK thought the Horizon Digital Economy Research Grants (EP/G065802/1, EP/M000877/1).

Open Access This article is distributed under the terms of the Creative Commons Attribution 4.0 International License (http://creativeco mmons.org/licenses/by/4.0/), which permits unrestricted use, distribution, and reproduction in any medium, provided you give appropriate credit to the original author(s) and the source, provide a link to the Creative Commons license, and indicate if changes were made.

\section{Appendix 1}

See Table 3. 


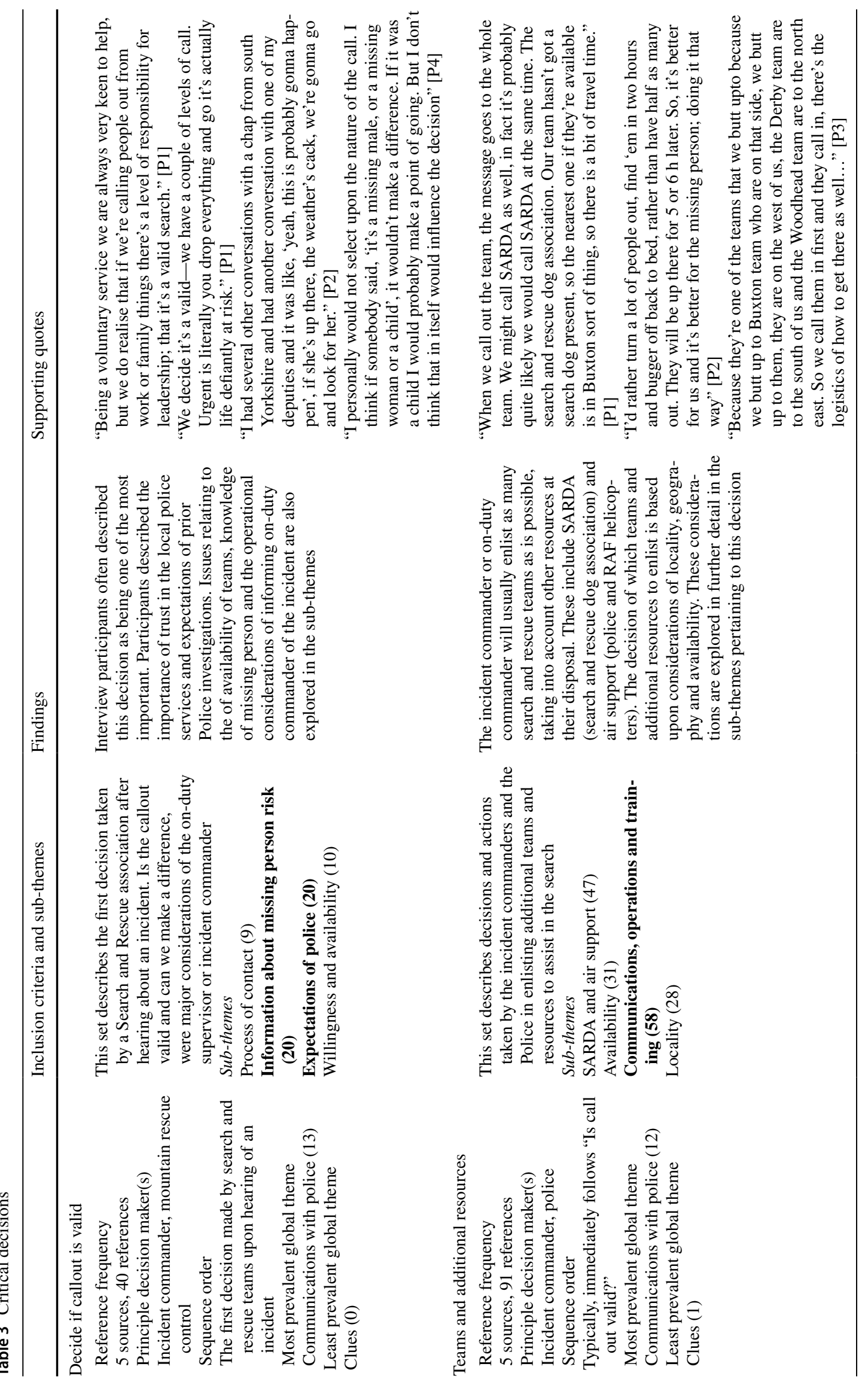




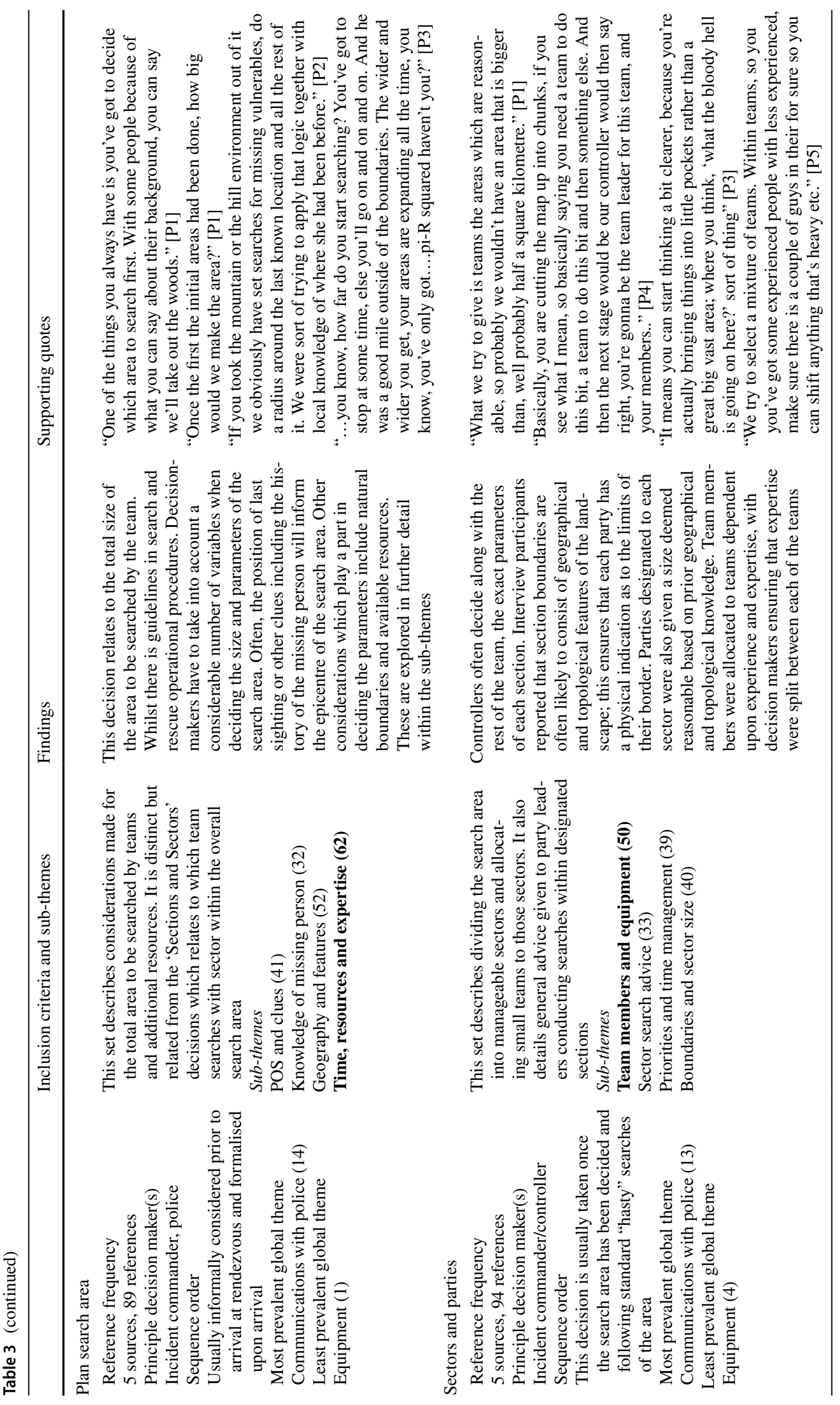




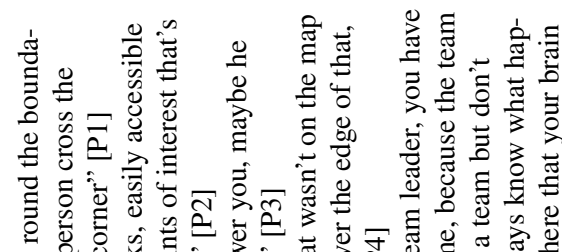

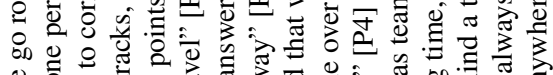

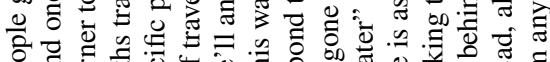

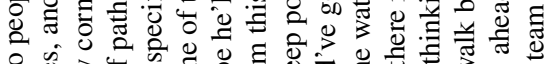

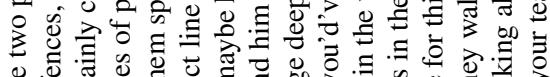

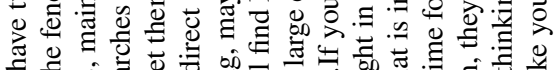

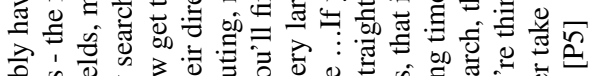

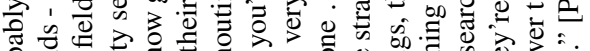

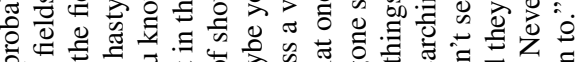

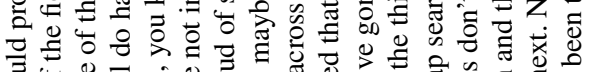

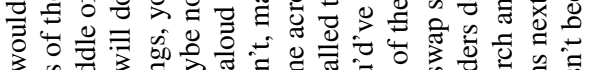

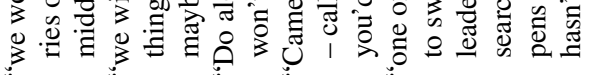

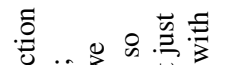

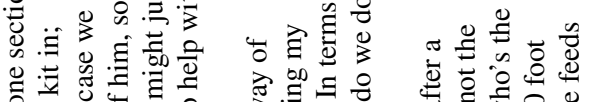

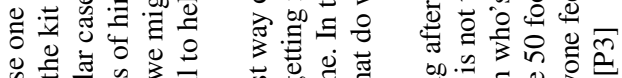

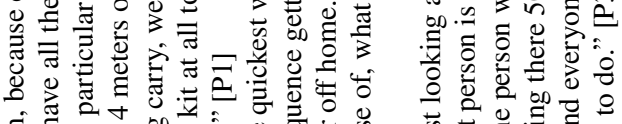

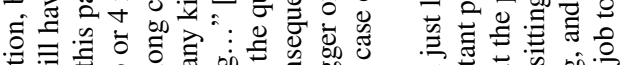

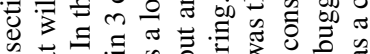

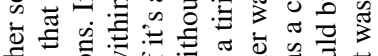

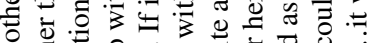

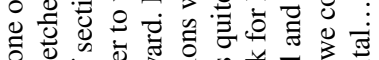

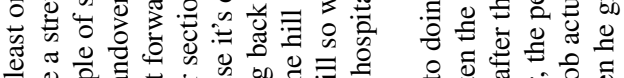

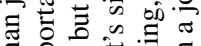
高矛卷

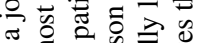
일

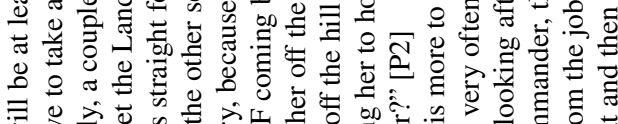

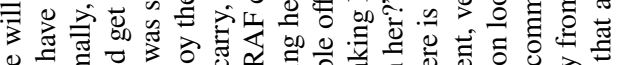

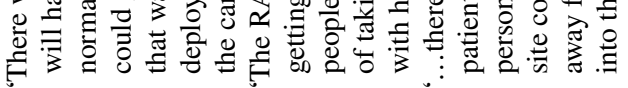

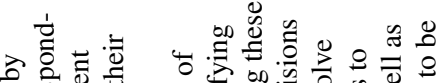

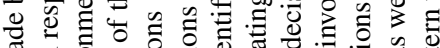

छ

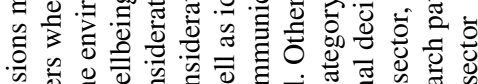

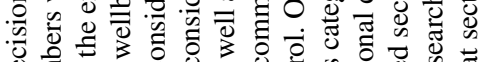

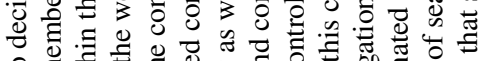

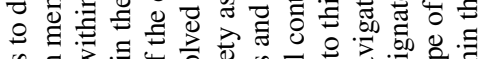

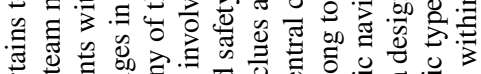

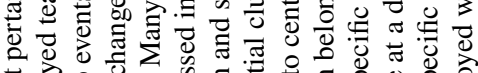

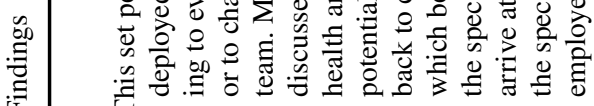

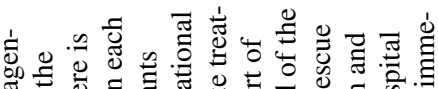

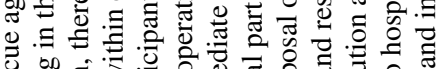

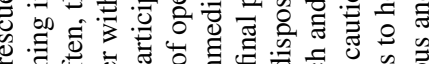

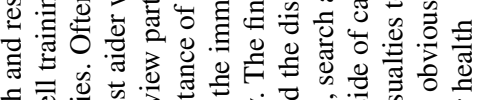

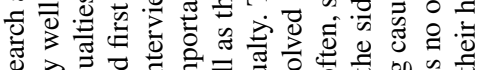
屯ํ.

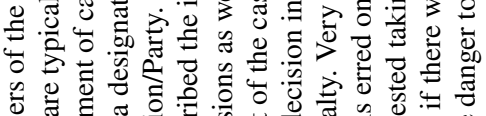

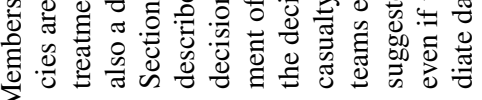

च

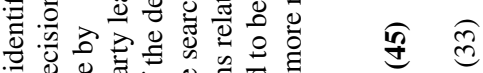

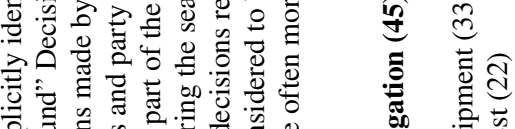

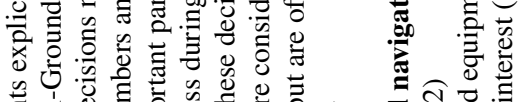

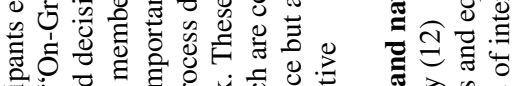

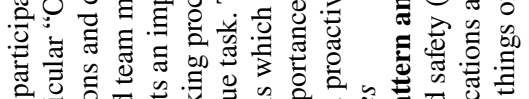

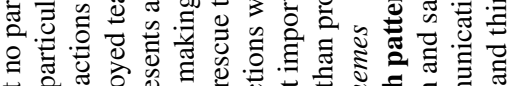

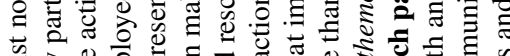

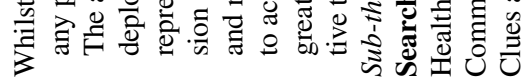

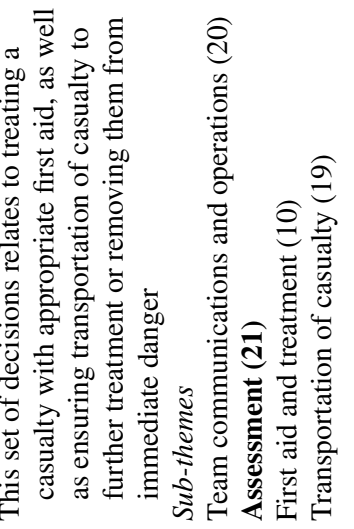
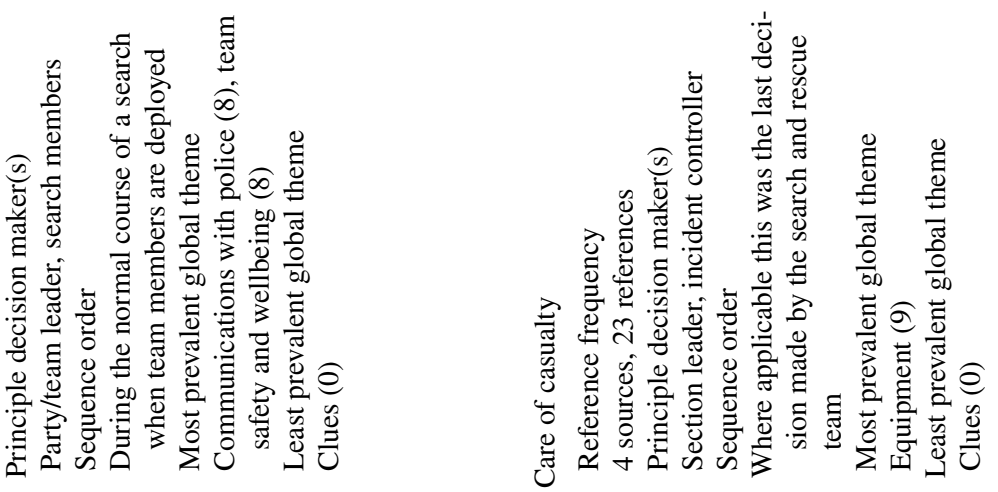

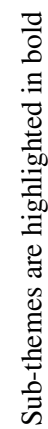




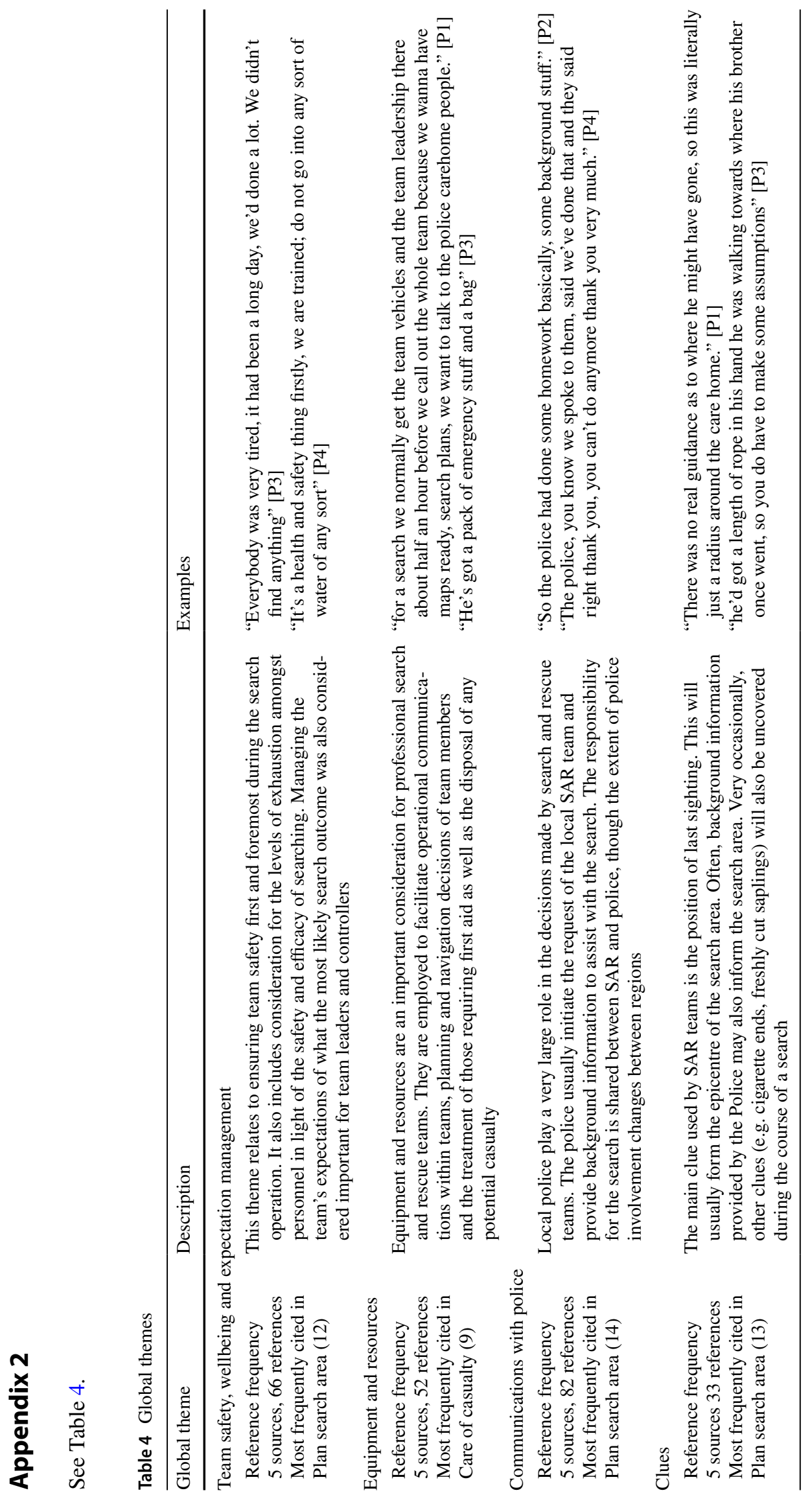




\section{References}

Adams JA, Cooper JL, Goodrich MA, Humphrey C, Quigley M, Buss BG, Morse BS (2007) Camera-equipped mini UAVs for wilderness search support: task analysis and lessons from field trials. J Field Robot 25:1-2

Biehal N, Mitchell F, Wade J (2003) Lost from view. The Policy Press, Bristol, Retrieved on September 172003

Braun V, Clarke V (2006) Using thematic analysis in psychology. Qual Res Psychol. https://doi.org/10.1191/1478088706qp063oa

Blandford A, Wong BLW (2004) Situation awareness in emergency medical dispatch. Int J Human Compt Studies 61 (4):421-452

Chaudet H, Pellegrin L, Bonnardel N (2015) Special issue on the 11th conference on naturalistic decision making. Cogn Technol Work 17(3):315-318. https://doi.org/10.1007/s10111-015-0330-6

Cooper DC, Jones A, Howells P, Jones C, Read A, O'Shea J, Evans J, Layland R (2008) Search Management. Mountain Rescue England and Wales, Torquay

Clark RE, Feldon D, van Merriënboer JJ, Yates K, Early S (2008) Cognitive task analysis. Handb Res Educ Commun Technol 3:577-593

Crandall B, Klein GA, Hoffman RR (2006) Working minds: a practitioner's guide to cognitive task analysis. Mit Press, Cambridge

Ellison A, Schindler I, Pattison LL, Milner AD (2004) An exploration of the role of the superior temporal gyrus in visual search and spatial perception using TMS. Brain 127(10):2307-2315

Flanagan JC (1954) The critical incident technique. Psychol Bull 51(4):327

Gigerenzer G, Goldstein DG (1996) Reasoning the fast and frugal way: models of bounded rationality. Psychol Rev 103(4):650-669. https ://doi.org/10.1037/0033-295X.103.4.650

Goldstein DG, Gigerenzer G (2002) Models of ecological rationality: the recognition heuristic. Psychol Rev 109(1):75-90. https://doi. org/10.1037/0033-295X.109.1.75

Kahneman DT, A (1979) Intuitive prediction: biases and corrective procedures. Manag Sci 12:313-327

Kahneman D, Tversky A (1979) Prospect theory: an analysis of decision under risk. Econometrica 47(2):263-291. https://doi. org/10.2307/1914185

Kiss M, Goolsby BA, Raymond JE, Shapiro KL, Silvert L, Nobre AC, Eimer M (2007) Efficient attentional selection predicts distractor devaluation: event-related potential evidence for a direct link between attention and emotion. J Cogn Neurosci 19(8):1316-1322

Klein GA (1993) A recognition-primed decision (RPD) model of rapid decision making. In: Klein GA, Orasanu J, Calderwood R, Zsambok CE (eds) Decision making in action: models and methods. Ablex Publishing Corporation, Norwood, pp 138-147

Klein GA, Calderwood R, Clinton-Cirocco A, Laboratory SR (1988) Rapid decision making on the fire ground. U.S. Army Research Institute for the Behavioral and Social Sciences, Arlington

Klein GA, Calderwood R, Macgregor D (1989) Critical decision method for eliciting knowledge. Syst Man Cybern IEEE Trans 19(3):462-472

Koester RJ, Stooksbury DE (1995) Behavioral profile of possible Alzheimer's disease patients in Virginia search and rescue incidents. Wilderness Environ Med 6(1):34-43

Koopman BO (1946) A theoretical basis for method of search and screening (No. CU-R-56) Columbia University, New York (Formerly classified US Military Document) Retrieved from. http:// www.dtic.mil/dtic/tr/fulltext/u2/214252.pdf

Lois J (2001) Managing emotions, intimacy, and relationships in a volunteer search and rescue group. J Contemp Ethnogr 30(2):131-179

Luini LP, Marucci FS (2015) Prediction-confirmation hypothesis and affective deflection model to account for split-second decisions and decision-making under pressure of proficient decision-makers. Cogn Technol Work 17(3):329-344. https://doi.org/10.1007/ s10111-015-0328-0

Militello LG, Hutton RJ (1998) Applied Cognitive Task Analysis (ACTA): a practitioner's toolkit for understanding cognitive task demands. Ergonomics 41(11):1618-1641

National Crime Agency (2016) UK Missing Persons Bureau:Missing Persons Data Report 2014/2015. London. Retrieved from http:// www.nationalcrimeagency.gov.uk/publications/696-missing-perso ns-data-report-2014-2015/file

O’Hare D, Wiggins M, Williams A, Wong W (1998) Cognitive task analyses for decision centred design and training. Ergonomics 41(11): 1698-1718

Okoli JO, Weller G, Watt J (2016) Information processing and intuitive decision-making on the fireground: towards a model of expert intuition. Cogn Technol Work 18(1):89-103. https://doi. org/10.1007/s10111-015-0348-9

Parr H, Stevenson O, Fyfe N, Woolnough P (2015) Living absence: the strange geographies of missing people. Environ Plan D Soc Space 33(2):191-208. https://doi.org/10.1068/d14080p

Prince M, Knapp M, Guerchet M, McCrone P, Prina M, Comas-Herrera A, Wittenberg R, Adelaja B, Hu B, King D, Rehill A and Salimkumar D (2014) Dementia UK report. Retrieved from.https://www. alzheimers.org.uk/sites/default/files/migrate/downloads/demen tia_uk_update.pdf

Perkins D (2013) Probability of Detection for the Search Manager. Centre for Search Research. Available at http://www.isarallian ce.com/general-library/download/68

Plant KL, Stanton NA (2016) Distributed cognition in search and rescue: loosely coupled tasks and tightly coupled roles. Ergonomics. https://doi.org/10.1080/00140139.2016.1143531

Rasmussen J (1997) Risk management in a dynamic society: a modelling problem. Saf Sci 27(2):183-213

Rogalski J (1999) Decision making and management of dynamic risk. Cogn Technol Work 1(4):247-256. https://doi.org/10.1007/s1011 10050021

Rowe M, Houston A, Molinari V, Bulat T, Bowen ME, Spring HBM (2015) The concept of missing incidents in persons with dementia. Healthcare 3(4):1121-1132. https://doi.org/10.3390/healthcare 3041121

Salas E, Klein GA (2001) Linking expertise and naturalistic decision making. Taylor \& Francis, London

Salmon P, Jenkins D, Stanton N, Walker G (2010) Hierarchical task analysis vs. cognitive work analysis: comparison of theory, methodology and contribution to system design. Theor Issues Ergon Sci 11(6):504-531

Salmon PM, Cornelissen M, Trotter MJ (2012) Systems-based accident analysis methods: a comparison of Accimap, HFACS, and STAMP. Saf Sci 50(4):1158-1170

Saunders B, Sim J, Kingstone T, Baker S, Waterfield J, Bartlam B, Jinks C (2018) Saturation in qualitative research: exploring its conceptualization and operationalization. Qual Quant 52(4):18931907. https://doi.org/10.1007/s11135-017-0574-8

Schraagen JM, Chipman SF, Shalin VL (2000) Cognitive task analysis. Lawrence Erlbaum Associates Publishers, New Jersey

Shepherd A (2000) Hierarchial task analysis. CRC Press, Boca Raton

Smith JF, Kida T (1991) Heuristics and biases: expertise and task realism in auditing. Psychol Bull 109(3):472-489. https://doi. org/10.1037/0033-2909.109.3.472

Stanton NA (2006) Hierarchical task analysis: developments, applications, and extensions. Appl Ergon 37(1):55-79. https://doi. org/10.1016/j.apergo.2005.06.003

Stevenson O, Parr H, Woolnough P, Fyfe N (2013) Geographies of missing people: processes, experiences, responses [online]. Project Report. University of Glasgow, Glasgow, UK. http://eprin ts.gla.ac.uk/81362/1/81362.pdf 
Thaler RH, Tversky A, Kahneman D, Schwartz A (1997) The effect of myopia and loss aversion on risk taking: an experimental test*. Q J Econ 112(2):647-661. https://doi.org/10.1162/003355397555226

Tversky A, Kahneman D (1973) Availability: a heuristic for judging frequency and probability. Cogn Psychol 5(2):207-232. https:// doi.org/10.1016/0010-0285(73)90033-9

van den Heuvel C, Alison L, Power N (2014) Coping with uncertainty: police strategies for resilient decision-making and action implementation. Cogn Technol Work 16(1):25-45. https://doi. org/10.1007/s10111-012-0241-8

Wilson JR, Corlett N (2005) Evaluation of human work. CRC Press, Boca Raton
Wolfe JM (1994) Guided search 2.0 a revised model of visual search. Psychon Bull Rev 1(2):202-238

Wong BLW, Blandford A (2002) Analysing ambulance dispatcher decision making: trialing emergent themes analysis. Proceedings of the HF2002 Human Factors Conference: Design for the whole person: integrating physical, cognitive and social aspects. Ergonomics Society of Australia, 2002

Zsambok CE, Klein GA (1997) Naturalistic decision making. L. Erlbaum Associates, Mahwah 